\title{
LONG-TERM NEARSHORE BATHYMETRY EVOLUTION FROM VIDEO IMAGERY: A CASE STUDY IN THE MIYAZAKI COAST
}

\author{
Haijiang Liu ${ }^{1,2}$, Makoto Arii ${ }^{1}$, Shinji Sato ${ }^{1}$ and Yoshimitsu Tajima ${ }^{1}$
}

\begin{abstract}
A four-year evaluation of the nearshore morphology based on video imagery was conducted in the Miyazaki coast, Japan. An automatic nearshore bathymetry mapping technique was developed based on the linear wave dispersion relationship, in which the wave phase velocity was estimated from the cross-correlation of neighboring pixels' brightness time series. Bathymetry estimation was carried out at both low and high tide levels of each day and integrated over a 7-day period which includes both the neap and spring tides. Model validation shows the present technique reproduced the ground truth data fairly well with a bias generally less than $0.3 \mathrm{~m}$ in the surf zone. In total, 88 bathymetry data were obtained within the investigation period. Subsequently, a two dimensional Empirical Orthogonal Function (EOF) analysis was applied to the time series of the obtained nearshore bathymetry data in order to investigate their long-term morphological behavior. Results from the first three modes of the EOF analysis represent the bathymetry variation from the nearshore response due to the cross-shore processes. The first mode reveals the coastal erosion occurred during the study period with a net loss of sand volume in the target region. Erosion was severe before December 2008, especially in the offshore region, and mitigated afterwards resulting a relatively equilibrium stage for the mean beach profile. The second mode shows the mean beach profile rotated in an anti-clockwise direction around a cross-shore location of $50 \mathrm{~m}$ away from the present shoreline, which led to a milder beach slope in the study period. The third mode represents the cross-shore movement of the longshore bar. Higher modes of the EOF analysis, such as fourth, fifth and sixth modes, correspond to the bathymetry variation due to the longshore sediment movement which frequently changed its direction as indicated from a number of nodal points in the relevant temporal eigenfunction distribution. Based on the EOF analysis, it is also confirmed that the nearshore morphological process in the target area was predominant by the cross-shore sand movement.
\end{abstract}

Keywords: long-term evolution; nearshore bathymetry mapping; video imagery; EOF analysis; cross-shore movement; longshore movement

\section{INTRODUCTION}

The nearshore, as a highly dynamic area, plays an important role in coastal studies. Bathymetry variation in the nearshore zone shows a wide range of spatial and temporal features, which embarrass the accurate evaluation on the morphological process. Traditionally, in-situ bathymetric survey methods, including the use of ships (the multi-beam sonar sounding system), amphibious vehicles or jet skis (various measuring equipments applying the acoustics approach in the frequency domain) and the remote-control boat, provide a large amount of excellent data at the specific time. However, these methods are, in general, inefficient and costly with a survey frequency only once or twice in one year, which are lack of spatiotemporal resolution to capture the bathymetric change on all interested space(meters to kilometers) and time-scales (hours to years). In addition, these techniques could not be applied under the large waves or strong current conditions, under which the significant bathymetry change undergoes and presents the most scientific interest.

In the last three decades, the remote sensing techniques are rapidly developed and implemented in the coastal engineering studies, and provide advanced opportunities for finding the physical insights of the nearshore hydro- and morpho-dynamics. Various remote sensing techniques have been applied to estimate the nearshore bathymetry. Bell (1999) mapped the wave celerity and direction from the standard marine X-band radar image sequences. Subsequently, by applying the linear wave theory, estimation on the nearshore bathymetry was conducted. Takewaka (2005) also measured the intertidal foreshore slopes with an X-band marine radar system. Meanwhile, Lyzenga (1985) well documented the application of the multispectral satellite imagery to the bathymetry estimation. Kanno et al. (2011) proposed a new method by combining the spatial interpolation and passive remote sensing to obtain the nearshore bathymetry information. In addition, Clarke and Werner (2003) applied the synoptic imaging for estimating various nearshore bathymetric patterns in the surf zone. In parallel, application of the shore-based video imagery to the nearshore morphology study becomes rather popular, especially with the worldwide use of the ARGUS system from 1980s (Holman and Stanley, 2007). The ARGUS research group has being working on the estimation of nearshore bathymetry or sand bar morphology

\footnotetext{
${ }^{1}$ Department of Civil Engineering, the University of Tokyo, 7-3-1 Hongo, Bunkyo-ku, Tokyo 113-8656, Japan.

${ }^{2}$ Department of Ocean Science and Engineering, Zhejiang University, 866 Yuhangtang Road, Hangzhou, Zhejiang, 310058, China.
} 
using video imagery for a long period (Lippmman and Holman, 1989; Plant and Holman, 1997; Stockdon and Holman, 2000; van Enckevort and Ruessink, 2001; Aarninkhof et al., 2003; Aarninkhof et al., 2005; Smith and Bryan, 2007; Dongeren et al., 2008). These studies mainly focused on the bathymetry estimation within a certain specified time period and at an assigned region, e.g., the intertidal zone. Additionally, Liu et al. (2008) carried out a 3-month monitoring on the morphodynamics of a river mouth sand spit to reveal the short-term coastal evolution processes induced by two typhoon events. Recently, Uunk et al. (2010), based on Aarninkhof et al. (2003), presents a fully automated procedure to derive the intertidal beach bathymetry in a 4 month period.

At the same time, long-term monitoring on the nearshore process is of great importance for the coastal region planning and management, as well as for predicting the future situation of the beach and coastline. Traditionally, this was done by the regular and monthly-to-yearly based field surveys, which are less of information on the temporal scale and heavily depended on the available survey data. The shore-based video system, however, makes a major contribution to this aim. The role of video imagery in predicting daily to monthly, seasonal to inter-annual coastal evolution, together with the beach recreation planning and coastal management, is demonstrated in various studies (Smit et al., 2007; Kroon et al., 2007; Jimenez et al., 2007; Pearre and Puleo, 2009; Liu et al., 2010). After gathering the long-term field data sets, a number of statistical techniques to analyze the long-term coastal morphological evolution are applied. Larson et al. (2003) reviewed various linear methods in data interpretation, e.g., encompassed bulk statistics, random sine functions, empirical orthogonal functions, canonical correlation analysis and principal oscillation pattern analysis. Southgate et al. (2003) summarized and assessed the nonlinear methods used in the coastal engineering study, including timedelay embedding technique, singular spectrum analysis, forecasting signatures, fractal analysis and neural networks. Recently, quite a few new approaches were developed to evaluate the coastal process, e.g., the non-linear principal component analysis by Ruessink et al. (2004), the behavioral template model by Davidson and Turner (2009), and the Bayesian network by Plant and Holland (2011). Up to now, long-term (seasonal to yearly) statistical analyses and assessment on the nearshore bathymetry derived from the shore-based video imagery are still limited.

In this study, a long-term evaluation of the nearshore morphological characteristics was conducted in the Miyazaki coast over a four-year period. The nearshore bathymetry was automatically derived from the video imagery based on the linear wave theory's dispersion relationship. Applying the crosscorrelation technique, the wave phase velocity was estimated from the brightness time series of four neighboring pixels. After obtaining the bathymetry data, an empirical orthogonal function analysis was applied to reveal the general feature and regularity of the nearshore morphological process in the target area. In the following section, the study area and field camera deployment are introduced, together with the recorded image properties. Then, the image process and the nearshore bathymetry estimation are specified step by step. The accuracy of the technique is also discussed. Subsequently, a long-term assessment on the nearshore morphology based on the empirical orthogonal function analysis is presented. Finally, a brief summary of the conclusions of this research is presented.

\section{STUDY AREA AND CAMERA DEPLOYMENT}

In this study, the Ooita coast area at the Miyazaki Prefecture, Japan, was investigated. The Ooita coast, as a part of the Miyazaki coast, is a steep sandy coast facing the Pacific Ocean and suffering from the serious coastal erosion in the last three decades. Various countermeasures have been implemented to mitigate the erosion problem in this region, e.g., seawall construction and beach nourishment (Liu et al., 2011). The Miyazaki coast characterizes with a neap/spring tidal range of $1 / 2$ $\mathrm{m}$ with a fortnight tidal period, and a significant offshore wave height generally less than $1 \mathrm{~m}$ with a significant wave period of 7-9 s.

In order to understand the nearshore hydrodynamic wave and current features, as well as the corresponding sediment movement behavior, a nearshore camera system was deployed in the study area from September 2006. The camera used in the field is the DualNight M12 type MOBOTIX network camera with a 450 wide angle lens and a physical focal length of $8 \mathrm{~mm}$. The camera was set on the top of a $10 \mathrm{~m}$ pole with a solar power supply. The daily image recording is hourly based from 7 am to $6 \mathrm{pm}$ (10 min recording at the beginning of each hour) with a sampling frequency of $2 \mathrm{~Hz}$. The recorded image is stored in a color JPEG format and has a resolution of $640 \times 480$ pixels. In this study, recorded images over a four year period from September 2006 to December 2010 were applied for data 
analysis. During this period, data deficiencies occurred nine times owing to the technical and mechanical problems. None of them is longer than two months.

\section{IMAGE ANALYSIS AND BATHYMETRY ESTIMATION}

\section{Image Analysis Procedure}

After the field recording, image analysis was conducted to extract the useful information for nearshore bathymetry estimation. Figure 1 shows the flow chart of image analysis used for the automatic bathymetry mapping, which includes 11 steps for achieving the local bathymetry data. In the first step, suitable images were automatically selected for further image process. An automatic approach for a long-term image analysis often meets the image quality problem owing to the hostile and uncontrollable field conditions (Guillen et al., 2008; Liu et al., 2009; Uunk et al., 2010). It occurs on days of low visibility, e.g., rain, fog or typhoon event, as well as owing to the pollution of camera lens cover. In addition, the excessive brightness caused by the strong sunlight reflection from the water surface at certain specific hours during some periods of the year can also be problematic. Here, the time-exposure and variance images were applied for image selection. The time-exposure image was applied to judge the image visibility, and the variance image was for analyzing wave conditions and breaking zone. Recorded images with large or low brightness intensity or over-violent waves were disregarded. In the automatic analysis procedure, recorded images around the high and low tide levels for each day were considered to estimate the corresponding bathymetry of that day. Selection of these two time periods can make good use of image information since they represents two extrema on the water level over one day. Considering that the wave breaking zone is a tough region for water depth estimation, variation of the wave breaking zone between the high tide and low tide levels in one day makes the bathymetry estimation become easy for the entire nearshore area under which two estimated results can complement with each other.

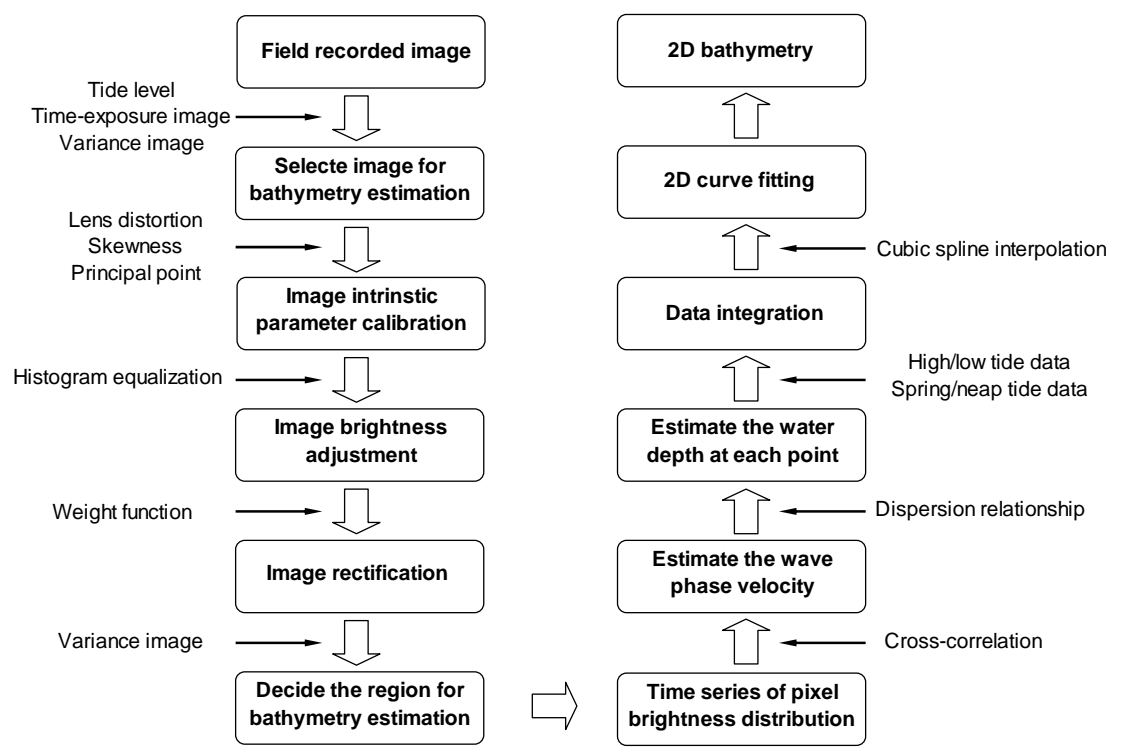

Figure 1. Image analysis flow chart for the nearshore bathymetry estimation.

\section{Intrinsic and Extrinsic Camera Calibration}

Intrinsic camera calibration was conducted to remove the distortion from camera physical defects. The intrinsic parameters reflect the physical characteristics of the lens, camera, and image acquisition hardware. The ARGUS group applied the photogrammetric theory proposed by Holland et al. (1997) for the intrinsic camera calibration. To calculate the intrinsic parameters, i.e., radial and tangential distortions, skewness coefficient and image principal point bias, a rather restricted setting for the camera and the planar pattern was needed in the classic ARGUS technique.

Later, Heikkila and Silven (1997), Zhang (2000) developed a considerably more flexible approach under which either the camera or the planner pattern can be freely moved without knowing the detailed motion. The proposed technique only requires the camera to observe a planar pattern, such as a checkerboard, shown at a few different orientations, and can be carried out even after the field camera 
installation. In their approach, they proposed the following camera matrix $K$ for the five intrinsic camera calibration parameters,

$$
K=\left[\begin{array}{ccc}
f_{c u} & \alpha_{c} f_{c u} & u_{0} \\
0 & f_{c v} & v_{0} \\
0 & 0 & 1
\end{array}\right]
$$

in which, $f_{c u}, f_{c v}$ is the focal length in pixels along the image $u$ and $v$ axis, respectively. The aspect ratio $f_{c v} / f_{c u}$ is different from unit if the pixels in image array are not square. Parameter $\alpha_{c}$ is the skewness coefficient defining the angle between the horizontal and vertical axes; $u_{0}, v_{0}$ are the image principal point coordinates. The distortion-free coordinates $x_{p}, y_{p}$ can be derived from the normalized point coordinates $x_{d}, y_{d}$ using the camera matrix,

$$
\left[\begin{array}{c}
x_{p} \\
y_{p} \\
1
\end{array}\right]=K\left[\begin{array}{c}
x_{d} \\
y_{d} \\
1
\end{array}\right]
$$

where the normalized coordinate is defined as follows,

$$
\left[\begin{array}{l}
x_{d} \\
y_{d}
\end{array}\right]=\left(1+k_{1} r^{2}+k_{2} r^{4}\right)\left[\begin{array}{l}
\frac{X_{c}}{Z_{c}} \\
\frac{Y_{c}}{Z_{c}}
\end{array}\right]+\left[\begin{array}{l}
2 k_{3} \frac{X_{c} Y_{c}}{Z_{c}^{2}}+k_{4}\left(r^{2}+2 \frac{X_{c}^{2}}{Z_{c}^{2}}\right) \\
k_{3}\left(r^{2}+2 \frac{Y_{c}^{2}}{Z_{c}^{2}}\right)+2 k_{4} \frac{X_{c} Y_{c}}{Z_{c}^{2}}
\end{array}\right]
$$

where $r^{2}=\left(X_{c}^{2}+Y_{c}^{2}\right) / Z_{c}^{2}$ and $\left[X_{c}, Y_{c}, Z_{c}\right]$ is the space coordinate in the camera reference frame. Parameters $k_{1}, k_{2}$ are the radial distortion coefficients and $k_{3}, k_{4}$ are the tangential distortion coefficients.

Figure 2 presents an example of the distortion-free snapshot image. Unrealistic warp of the wave crest lines, especially near the image margin, was corrected in the distortion-free image. Visualization of the complete lens distortion obtained in Fig. 2 is illustrated in Fig. 3. Effect from the lens distortion becomes significant with the distance away from the image center. The effective displacement induced by the lens distortion at the upper-right corner of the image is even larger than 20 pixels. The radial component of the image distortion model plays a much more influential role than that of the tangential component. It is also observed that the distorted image center is different from the location of the principal point with a horizontal/vertical bias of $-15 /+3$ pixels, respectively. All intrinsic camera calibration parameters for field-deployed MOBOTIX M12 camera with an $8 \mathrm{~mm}$ lens are tabulated in Table 1.

After obtaining the distortion-free image, the RGB colored image was converted to the grayscale image for further analyses. In addition, image brightness intensity was also adjusted to improve the image contrast for spreading the intensity values over the full available range, i.e., [0 255], by the image histogram equalization.

Subsequently, the extrinsic camera calibration was carried out which comprises the geometrical description of the camera position and orientation relative to the ground coordinate system. Image rectification was conducted to acquire the calibration parameters which link the accurate georeferencing (the real world coordinates, $x, y, z$ ) with the oblique video images (the image coordinate, $u$, $v$ ). This procedure is crucial for the concrete discussion on the nearshore process. After the laboratory calibration for intrinsic camera parameters, Holland et al. (1997) conducted the field calibration to determine extrinsic parameters on the basis of the derived geometrical relationship between the image and ground coordinates. Based on this approach, Liu et al. (2008) introduced the weight function to the ground control points (GCP) to improve the accuracy of image rectification by enhancing the role of camera-far-field GCPs in the least-square analysis. This is ascribed to the fact that different pixels in the obliquely recorded image play different roles in terms of image rectification with the camera-farfield GCPs being more important considering that the image spatial resolution over there is relatively 
low. Image rectification is performed to a horizontal plane at the temporal tidal level. An example of the geo-rectified image on Feb 4, 2008, is shown in Figure 4(a).

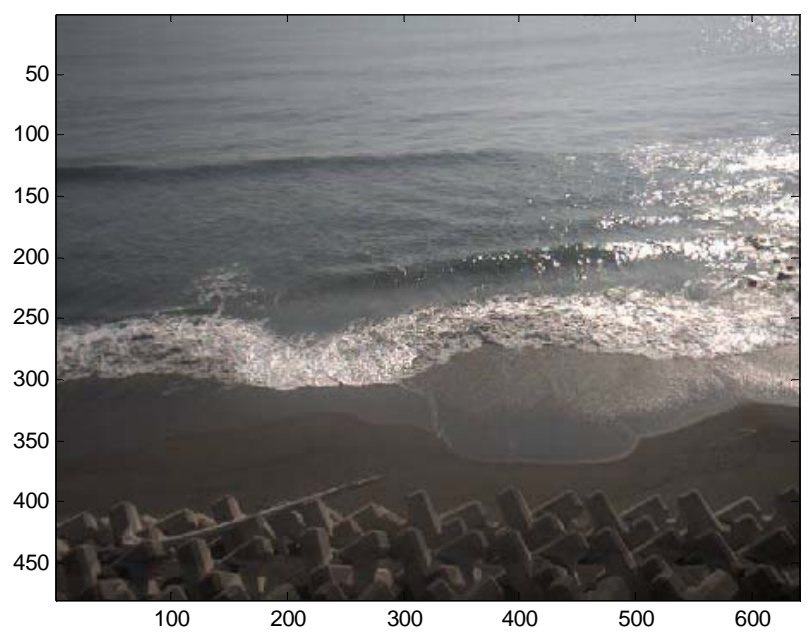

Figure 2. Undistorted image after the intrinsic camera calibration.

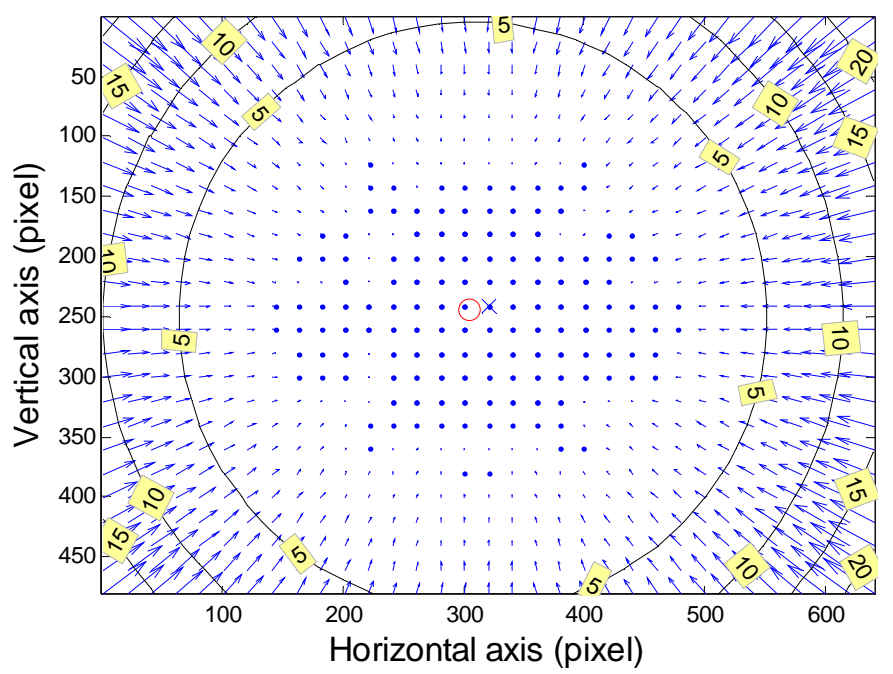

Figure 3. Complete distortion model for the МОВОтІX M12 network camera with an $8 \mathrm{~mm}$ lens, including both the tangential and radial components of the image distortion model. Mark ' $x$ ' indicates the distorted image center and mark ' $o$ ' indicates the correct image principal point location. Each arrow represents the effective displacement of the corresponding pixel induced by the lens distortion. Contour lines represent the isolines of pixel displacement by the lens distortion.

\begin{tabular}{|} 
Table 1. The intrinsic camera calibration parameters fro the MOBOTIX M12 network camera \\
with an 8 mm lens used in the field. \\
\hline$f_{c u}$ & $f_{c v}$ & $a_{c}$ & $u_{0}$ & $v_{0}$ & $k_{1}$ & $k_{2}$ & $k_{3}$ & $k_{4}$ \\
\hline 789.39 & 791.53 & $-6.9 \mathrm{e}-4$ & 304.69 & 242.86 & -0.21 & -0.04 & $1.8 \mathrm{e}-3$ & $9.2 \mathrm{e}-4$ \\
\hline
\end{tabular}

\section{Wave Phase Velocity Estimation}

After obtaining the geo-rectified image, the region of interest was specified for bathymetry estimation, which covers a cross-shore distance from the averaged shoreline to the area beyond the breaking zone. Bathymetry estimation was not conducted in the vicinity of the wave breaker where the persistent white foam caused by the wave breaking can mislead the wave celerity estimation. This procedure was implemented after the variance image. Then, the wave phase velocity was estimated based on the cross-correlation approach in a horizontal two-dimensional viewpoint. To estimate the wave celerity at a specified location, four neighboring points along the horizontal and vertical directions with a coordinate interval of 6 pixels each other (corresponding to a $6 \mathrm{~m}$ spatial interval in 
reality) were selected as shown in Fig. 4(a) for points P1-P4. Cross-correlation is calculated based on the time series of the brightness intensities at these four points (Fig. 4b). A time series lasting for $150 \mathrm{~s}$ was applied to cover an enough long time duration and avoid the error induced by the abrupt brightness fluctuation in the recorded images owing to the instantaneous cloud coverage. A time duration corresponding to the maximum cross-correlation value is assumed to be time interval for wave passing through the relevant two points. Then, the wave phase velocity is calculated. Subsequently, water depth at this point can be derived from the linear wave theory's dispersion relationship,

$$
h=\frac{1}{k} \operatorname{atanh}\left(\frac{C^{2} k}{g}\right)
$$

in which, $h$ is the water depth, $g$ is the gravitational acceleration, $k$ is the wave number and $C$ is the wave phase velocity.

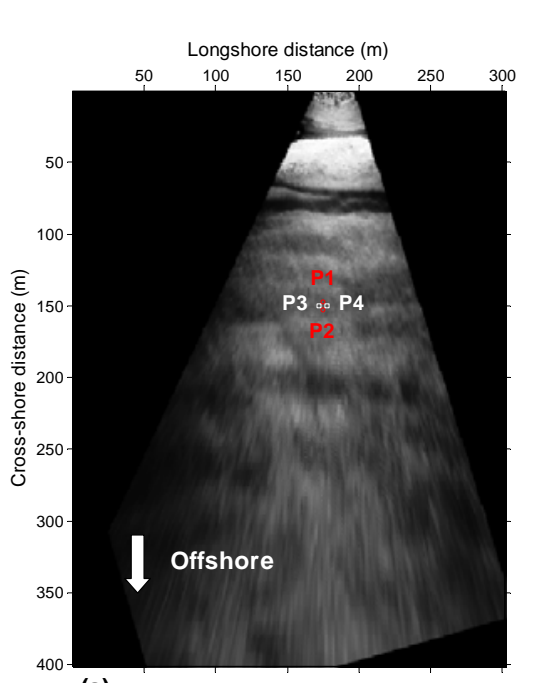

(a)
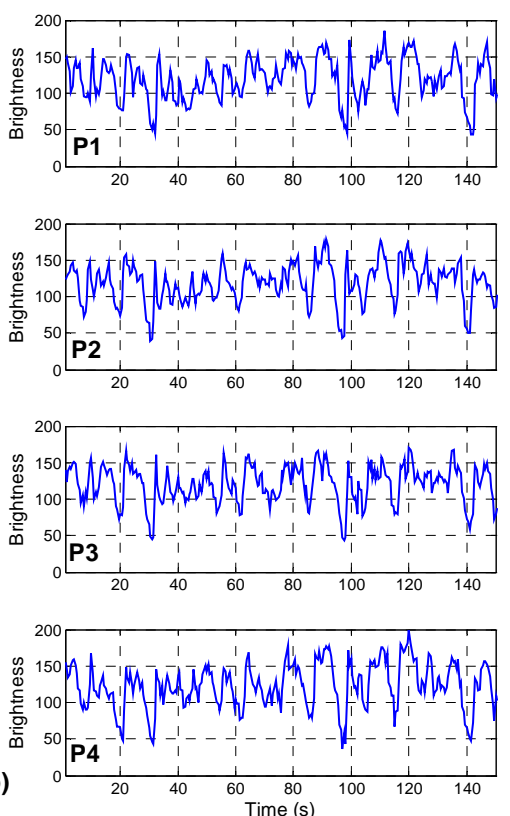

Figure 4. (a) A geo-rectified image on Feb 4, 2008. (b) Time series of brightness intensities at four neighboring pixel points, P1, P2, P3 and P4 indicated in (a).

Applying the same technique to the whole nearshore area with a cross-shore spatial interval of $3 \mathrm{~m}$ and a longshore spatial interval of $5 \mathrm{~m}$ (taking into account that the cross-shore bathymetry variation is more prevalent and the bathymetry in the longshore direction is relatively uniform), a horizontal twodimensional nearshore bathymetry can be estimated after adjusting with the concurrent tide information. In this paper, the bathymetry data after the tide level adjustment are all based on the Tokyo Peil (T.P.) standard. T.P. zero corresponds to the average water level at the Tokyo Bay, which is a standard geodetic datum used in Japan. In this study, an integrated nearshore bathymetry over a 7day period was regarded as the representative sub-aquatic topography within this time duration. A 7day criterion was selected based on the local fortnight tide period under which the data analysis covers images recorded during both the spring tide and the neap tide, which can compensate the bathymetry estimation among various tidal levels. As a result, 14 data can be obtained for each location during the 7-day estimation in case of the successive and suitable image conditions (2 data per day for the high and low tide, respectively). Nevertheless, if the estimated data set is less than 7 (half of the total expected number), bathymetry estimation for the relevant week will be disregarded. As for the remaining cases and at a specified location, estimated water depth with a significant bias was also removed in terms of the local data standard derivation before further data integration. An averaged value of the remaining data was finally assumed to be the water depth of the specified location in the relevant period.

\section{Estimated Nearshore Bathymetry}

Figure 5(a) presents an example of the estimated nearshore bathymetry from one week video images recorded starting from Aug 18, 2008. The blank area corresponds to the region that was not covered by the camera image. Bathymetry estimation was conducted in a trapezoid area starting at the 
mean shoreline with a longshore span of $40 \mathrm{~m}$, extending to the $140 \mathrm{~m}$ offshore where it spreads to 140 $\mathrm{m}$ in the longshore direction. Because the bathymetry estimation from the wave phase velocity cannot be implemented for all designed points due to the aforementioned image problems, a 2-D curve fitting using the cubic spline interpolation and extrapolation was applied to obtain the bathymetry values at missing points in the whole region of interest. Therefore, unrealistic data integration may be introduced, especially in the case of data extrapolation. Data extrapolation was mainly implemented near the shoreline where significant wave uprush and downwash processes make the image-based bathymetry derivation impossible. In the camera-far field where the image spatial resolution is low, correct wave celerity estimation becomes difficult at some locations where the bathymetry values were also estimated from the extrapolation of neighboring data. Nevertheless, as shown in Fig. 5(a), estimated nearshore bathymetry demonstrates the general decreasing feature along the cross-shore direction far away from the shoreline, as well as the heterogeneous 3D bathymetry pattern in the longshore direction.
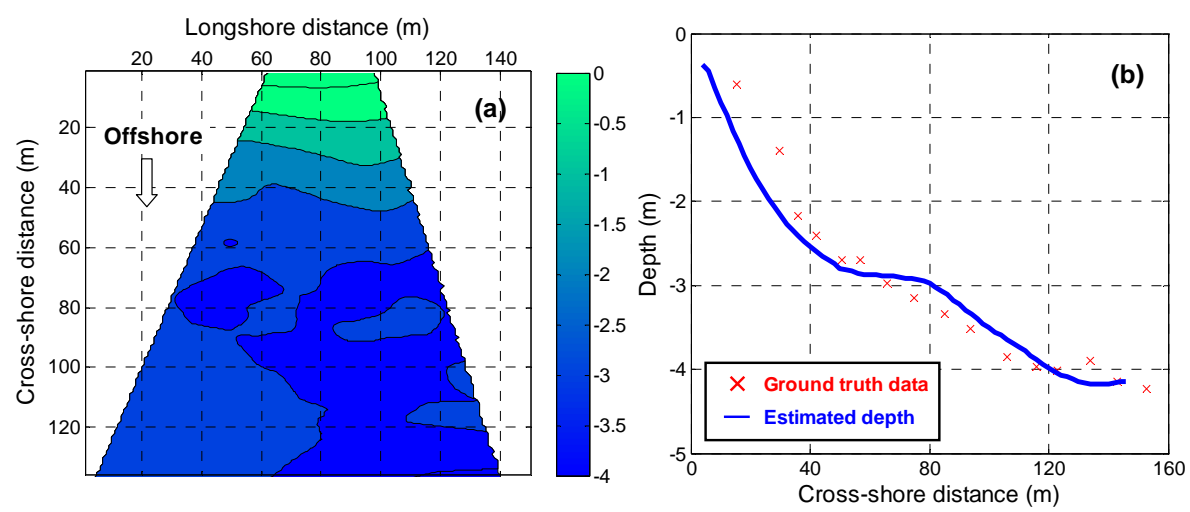

Figure 5 (a) An example of the estimated nearshore bathymetry from the recorded images for the week of Aug 18, 2008. (b) Comparison between the measured and the estimated bathymetry data along a cross-shore section at a longshore location of $90 \mathrm{~m}$ for the week of Dec 15, 2010.

Applying this technique 88 bathymetry data, in total, were derived within the period from September 2006 to December 2010 with a data sampling frequency of every two or three weeks. Comparing to the alternative direct measurement approaches which are, in general, annually based, it significantly improves the temporal and spatial resolution on the nearshore bathymetry survey. At the same time, such technique is also fairly cost-efficient as pointed out by Aarninkhof et al. (2005). This technique can also directly apply to the surf zone where the dynamic feature is prominent and important for the nearshore morphology. In the surf zone, traditional methods can not be deployed easily due to the hostile natural condition. Based on the automatic bathymetry mapping technique presented here, a large amount of nearshore bathymetry datasets was obtained which makes the precise long-term evaluation become feasible and reliable.

\section{Validation}

Validation of the present automatic bathymetry mapping technique was conducted by comparing the image-derived water depth to the in-situ measured data. Result is illustrated in Fig. 5(b). Bathymetry survey was performed annually in the target area using ships with the multi-beam sonar sounding system. However, data cannot be collected at the nearshore area owing to the severe wave conditions. To achieve the correct depth data in this region, a jet ski with a depth measuring facility was deployed in a ground truth testing to survey the sub-aquatic topography data on December 15 , 2010. In general, the estimated water depth matches the ground truth data fairly well with a bias less than $0.3 \mathrm{~m}$. A large discrepancy was observed near the shoreline with a maximum overestimation being $0.7 \mathrm{~m}$. The estimated profile is prone to an unrealistic deepening in this region. In the present analysis, image rectification was carried out to a concurrent tide level, which neglects the influence from the increasing wave height (shoaling effect) due to the wave nonlinearity, as well as the mean water level change owing to the wave set-up. This results the real field wave feature at a high elevation to be projected and rectified to a lower level, i.e., the instantaneous tide level, which induces an overestimation on the wave celerity, and induces a relatively large water depth. Applying the linear dispersion relationship, Stockdon and Holman (2000) showed in their study, differences between estimated and observed depths are more than $1 \mathrm{~m}$ in depths less than 3 or $4 \mathrm{~m}$ although their estimation presents a good result for the area seaward of the breaker zone. They attribute these errors to the wave nonlinearities and difference in optical signatures of breaking and non-breaking waves in the area 
further onshore. Aarninkhof et al. (2005) also argued the similar problem in their sub-tidal bathymetry estimation based on time-exposure video images after considering the wave energy dissipation.

In the nearshore, applying the first-order linear dispersion relationship induces inaccuracies in estimating the phase velocity. Horikawa and Kuo (1966) proposed the wave phase velocity based on the second-order solitary wave theory as,

$$
C=\sqrt{g(h+H)}
$$

where $H$ is the wave height. The solitary wave theory is assumed to be more appropriate and accurate within the surf zone (Thornton and Guza, 1982). The potential errors (depth overestimation) associated with inferring water depths from the linear theory rather than the finite amplitude dispersion theory can be as much as $42 \%$ (Stockdon and Holman, 2000).

\section{LONG-TERM EVALUATION OF THE NEARSHORE MORPHOLOGY}

\section{Empirical Orthogonal Function Analysis}

A long-term assessment of the nearshore sediment movement behavior in the target area was conducted based on the Empirical Orthogonal Function (EOF) analysis, which is also referred to as the Principal Component Analysis (PCA). The EOF technique is a statistical decomposition technique developed to separate the temporal and spatial dependence of the original dataset.

The application of EOF technique to the coastal study starts from the mid 1970s. Pioneer works have been conducted by Winant et al. (1975) to describe the beach profile changes and by Aubrey (1979) to study the seasonal patterns of on/offshore sediment movement. These studies, focusing on the cross-shore variability, relate the first three eigenfunctions to the variation in profile configuration representing the mean beach profile, the seasonal winter bar and summer berm profiles, and the low tide terrace. Recently, EOF has become a powerful and increasingly popular data analysis tool that is routinely applied to study shoreline and beach profile evolution in the coastal environment. Pruszak (1993) analyzed the local cross-shore feature of the beach profile on the basis of the EOF analysis. Miller and Dean (2007) applied the EOF technique to the assessment on the longshore shoreline variability. Fairley et al. (2009) used it to identify and characterize the shoreline changes behind detached breakwaters. At the same time, such data-driven model was also used for the forecast of sandbank evolution (Reeve et al., 2008).

In general, the aforementioned studies determined the eigenfunctions of the variations in seabed level or shoreline position in time along a fixed cross-shore or longshore transect. EOF analysis operates by exploiting the properties of matrices to identify patterns of standing oscillations within a data set which can then be used to represent the data in a more compact form. It reduces the number of data variables required to represent a specified data set. As for the 1-D EOF analysis, this is achieved by linearly decomposing a data set in terms of orthogonal functions both in space and time (Aubrey, 1979; Miller and Dean, 2007),

$$
h(x, t)=\sum_{m=1}^{n} h_{m}(x, t)=\sum_{m=1}^{n} a_{m} c_{m}(t) e_{m}(x)
$$

where $h(x, t)$ is the original bathymetry data or shoreline position recorded at any cross-shore or longshore location $x$ for any time $t$ in which each row contains a series of temporal observations at a specified location and each column includes the spatial observations at a specified time. The variable $h_{m}(x, t)$ is the $m$-th combined eigenfunction pair which consists of the $m$-th spatial eigenfunction, $e_{m}(x)$, and the $m$-th temporal eigenfunction, $c_{m}(t)$. Parameter $n=\min \left(n_{x}, n_{t}\right)$, where $n_{x}$ and $n_{t}$ are the number of spatial and temporal samples, respectively. The normalizing factors $a_{m}$ are determined by,

$$
a_{m}=\sqrt{\lambda_{m} n_{x} n_{t}}
$$

in which $\lambda_{m}$ is the eigenvalue associated with the $m$-th eigenfunction. The spatial eigenfunctions, $e_{m}(x)$, are simply the eigenfunctions (eigenvectors) associated with the spatial covariance matrix and can be decided from the equation, 


$$
\mathbf{A} e_{m}(x)=\lambda_{m} e_{m}(x)
$$

where the symmetric correlation matrix $\mathbf{A}$, or sum of squares and cross products of the data matrix, is formed by,

$$
\mathbf{A}=\frac{1}{n_{x} n_{t}}\left(\mathbf{H H}^{T}\right)
$$

in which matrix $\mathbf{H}$ is made up of the original dataset $h(x, t)$ and $T$ is the matrix transpose operator. Similarly, the temporal eigenfunctions, $c_{m}(t)$, are found by equations,

$$
\begin{aligned}
& \mathbf{B} c_{m}(t)=\lambda_{m} c_{m}(t) \\
& \mathbf{B}=\frac{1}{n_{x} n_{t}}\left(\mathbf{H}^{T} \mathbf{H}\right)
\end{aligned}
$$

The matrices $\mathbf{A}$ and $\mathbf{B}$ have the same non-trivial eigenvalues. The eigenfunction associated with the largest eigenvalue represents the bulk of the variability in the dataset, or the best-fit data in a least squares sense; whereas, each subsequent eigenfunction accounts for the majority of the remaining data variability. Therefore, the original data set can be efficiently represented by a few empirical functions (the lower order modes) which extract the dominant and meaningful signals from the background noise described by the higher order modes.

In this study, a two-dimensional EOF analysis was required to capture the horizontal twodimensional nearshore morphological pattern in both cross-shore and longshore directions. There are several ways in which the horizontal 2-D data may be expanded in terms of eigenfunctions with respect to the discrete nearshore bathymetry data, $h\left(x_{i}, y_{j}, t_{k}\right)$. The first method was described by Uda and Hashimoto (1982),

$$
h\left(x_{i}, y_{j}, t_{k}\right)=\sum_{m=1}^{n} e_{m}\left(x_{i}, t_{k}\right) f_{m}\left(y_{j}, t_{k}\right)
$$

where $e_{m}\left(x_{i}, t_{k}\right)$ is the longshore eigenfunctions and $f_{m}\left(y_{j}, t_{k}\right)$ are the cross-shore eigenfunctions. Both eigenfunctions are time-dependent at this stage. Subsequently, the eigenfunctions $e_{m}$ and $f_{m}$ can, themselves, be decomposed into a form exactly analogous to the case in Eq. (6).

In addition, an alternative decomposition process has been proposed by Hsu et al. (1994),

$$
h\left(x_{i}, y_{j}, t_{k}\right)=\sum_{m=1}^{n} e_{m}\left(x_{i}\right) f_{m}\left(y_{j}\right) c_{m}\left(t_{k}\right)
$$

in which the longshore eigenfunctions $e_{m}\left(x_{i}\right)$, the cross-shore eigenfunctions $f_{m}\left(y_{j}\right)$, and the temporal eigenfunctions $C_{m}\left(t_{k}\right)$ are completely separated.

The third approach was suggested by Reeve et al. (2001) in which the two-dimensional data grid of points is treated at as a large one-dimensional data set, and use Eq. (6) with spatial eigenfunction $e_{m}$ covering all combinations of $\left(x_{i}, y_{j}\right)$. The element $a_{m n}$ in symmetric correlation matrix $\mathbf{A}$ represents the temporal correlation between a selected location (denoted by the $m$ subscript) and that at other nearshore location (denoted by the $n$ subscript). Afterwards, the eigenfunctions proceed as in the 1-D case. The spatial structure of each spatial eigenfunction can, then, be retrieved by noting the correspondence between $e_{m}$ and $\left(x_{i}, y_{j}\right)$. In fact, this is a quasi-2D EOF analysis method based on the 1D EOF technique. Reeve et al. claimed that this method is more straightforward than other 2D methods and is easy for result interpretation. This procedure does not need a priori assumption of separability of cross-shore and longshore dependence. Considering the target region in this study exhibiting strong joint cross-shore and longshore variability, as well as the unequal number of bathymetry data along the cross-shore and longshore directions due to the trapezoid shape of image recording as shown in Fig. 5(a), the Reeve's method for the 2D EOF analysis was applied. 


\section{Result and Discussion}

Table 2 summarizes the results from the EOF analysis. It demonstrates that $99.44 \%$ of the mean square value of the data is contained in the first mode and the first 6 eigenfunctions capture $99.85 \%$ of the mean square value of the data, which is equal to the trace, i.e., sum of the diagonal elements of the symmetric correlation matrix $\mathbf{A}$ or $\mathbf{B}$ in Eqs. (9-10). The mean square of the data is the average of the square of the all nearshore bathymetry data corresponding to the entire 88 image-derived datasets. In the present analysis, the datasets were not demeaned prior to subjecting them to the EOF analysis, the first spatial eigenfunction therefore typically bears a strong resemblance to the statistical mean, or corresponds to the mean bathymetry over the research period (Reeve et al., 2001; Miller and Dean, 2007, Liu et al., 2010). The higher modes' eigenfunctions represent the variability about the mean bathymetry under which subsequent modes are responsible for an increasingly smaller percentage of the total variability. In this case, the second to sixth eigenfunctions together account for $73.5 \%$ of the total variance about the mean bathymetry with a largest value of $39.9 \%$ for the second mode and gradually decreasing to $5.6 \%$ for the sixth mode. Further higher modes represent minimal amount of variability. A value of $73.5 \%$ corresponds to a large proportion of the data variance for such statistical analysis (Reeve et al., 2001). It suggests that the EOF approach can describe the variability of the nearshore bathymetry data fairly efficiently.

\begin{tabular}{|c|c|c|c|c|}
\hline \multicolumn{5}{|c|}{ Table 2. The results of the EOF analysis. } \\
\hline Eigenfunction mode & $\begin{array}{c}\text { Normalized } \\
\text { Eigenvalue }\end{array}$ & $\begin{array}{c}\text { \% Mean square } \\
\text { (cumulative) }\end{array}$ & \% Variance & $\begin{array}{c}\text { \% Variance } \\
\text { (cumulative) }\end{array}$ \\
\hline 1 & 0.994395 & 99.44 & - & - \\
\hline 2 & 0.002239 & 99.66 & 39.9 & 39.9 \\
\hline 3 & 0.000837 & 99.75 & 15.0 & 54.9 \\
\hline 4 & 0.000398 & 99.79 & 7.1 & 62.0 \\
\hline 5 & 0.000330 & 99.82 & 5.9 & 67.9 \\
\hline 6 & 0.000315 & 99.85 & 5.6 & 73.5 \\
\hline
\end{tabular}

Figure 6 shows the assessment of nearshore bathymetry characteristics based on the first three modes of the EOF analysis. Since there are no physical assumptions made in the EOF analysis, the resulting eigenfunctions are purely mathematical in nature and do not have inherent physical insights. Interpretation of the derived eigenfunctions is rather straightforward. Figures 6(a) is the spatial eigenfunction distribution from the first mode EOF analysis, which presents a uniform feature along the longshore direction and a decreasing trend from the land to sea in the cross-shore direction (being aware that the corresponding temporal eigenfunction takes negative values). As aforementioned, this spatial eigenfunction distribution is quite similar to the mean bathymetry data within this 4-year study period. All positive values are confirmed for the first mode spatial eigenfunction, this indicates that the spatial variation is in phase for the entire area, and is more reflective of typical nearshore response due to the cross-shore processes. At the same time, all negative values are confirmed for the first mode temporal eigenfunction from the EOF analysis as shown in Fig. 6(b). Although with certain fluctuation, a decreasing trend of the temporal eigenfunction (a thick dashed line in Fig. 6b) demonstrates an erosion tendency in this area over the research period. A net reduction of the entire nearshore sand volume can be estimated according to the temporal eigenfunction distribution. The mean cross-shore beach profile erosion process can be identified into two stages separating at the time of Dec 2008. Before it, coastal erosion is significant with a rate of $6.67 \%$ per year in terms of the sand volume. Nevertheless, after Dec 2008, the erosion process is considerably mitigated and the nearshore bathymetry generally undergoes an equilibrium state. Based on the first mode EOF analysis, nearshore morphological change due to the cross-shore sand movement is schematically illustrated in Fig. 7(a). Large spatial eigenfunction values in the offshore area (Fig. 6a) demonstrate the bathymetry variability is more significant, or the erosion of the mean beach profile is more severe in the offshore region as indicated with two non-uniform arrows in Fig. 7(a). 

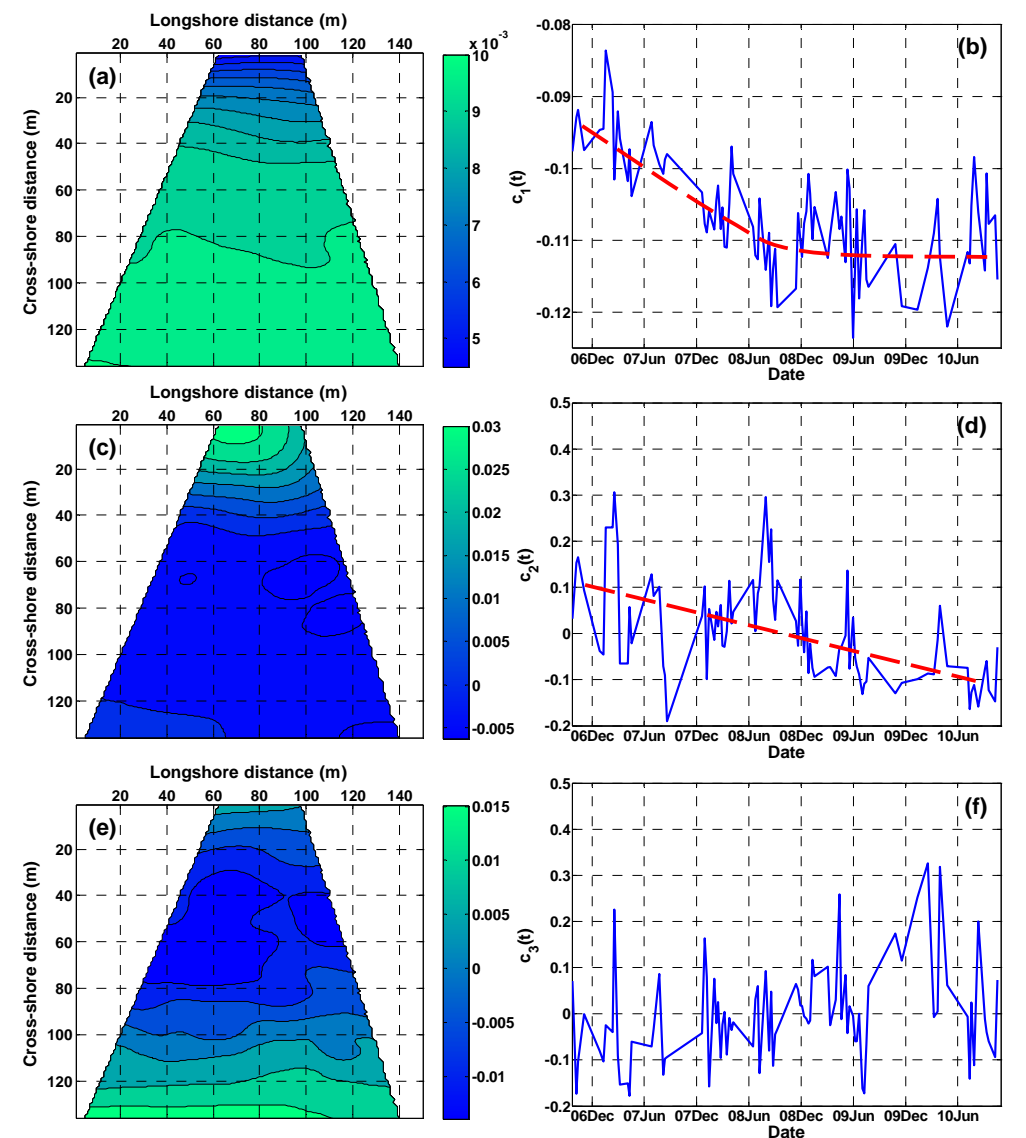

Figure 6. Evaluation of the nearshore bathymetry characteristics based on the first three modes of the EOF analysis. Upper panel: first mode functions; middle panel: second mode functions; Lower panel: third mode functions. Left panel: Contour plot of the spatial eigenfunctions; Right panel: time series of the temporal eigenfunctions. Thick dashed lines in (b) and (d) represent the trend lines of the relevant temporal eigenfunctions.

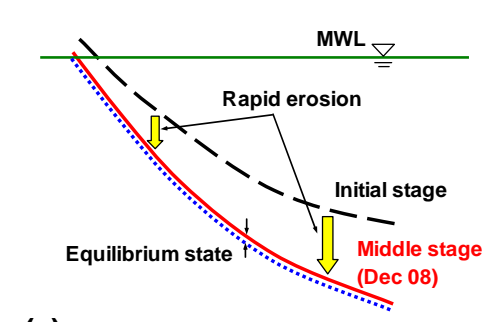

(a)

Final stage

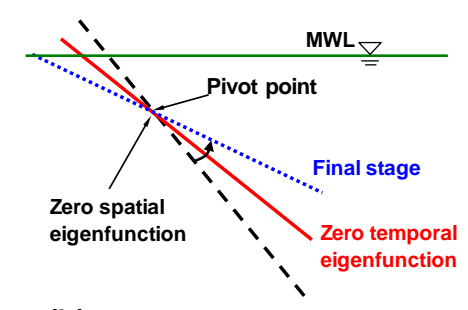

(b)

Initial stage

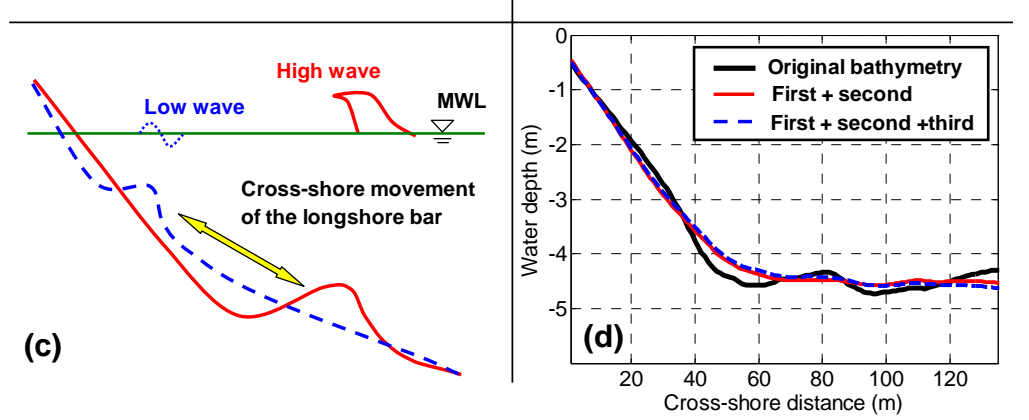

Figure 7. (a-c) Schematic diagram of the nearshore bathymetry variation represented from the first mode (a), the second mode (b) and the third mode (c) of the EOF analysis. (d) Comparison among the original bathymetry data, the EOF's first two modes result and the EOF's first three mode result along a cross-shore section at a longshore location of $80 \mathrm{~m}$ for the week of Feb 10, 2007. 
Results from the second mode of the EOF analysis are illustrated in Figs. 6(c) and 6(d) with respect to the spatial and temporal eigenfunctions, respectively. A relatively uniform pattern in the longshore direction is observed regarding to the spatial eigenfunction distribution. Hence, the second mode results also correspond to the cross-shore beach profile variation. A zero value of the spatial eigenfunction at the cross-shore location about $50 \mathrm{~m}$ indicates the nearshore bathymetry is stable and time-independent at this point. However, away from this point, positive and negative values of the spatial eigenfunction are observed on two sides which suggest the nearshore morphology is out of phase in these two areas. This point separates adjacent areas of erosion and accretion. Taking into account the temporal eigenfunction distribution in Fig. 6(d), although fluctuated, a general decreasing trend from a positive to a negative value can be drawn as highlighted using a thick dashed line. This line has one nodal point passing through the zero temporal eigenfunction around Dec 2008. Considering that the second mode spatial eigenfunction represents a cross-shore process, variation in the second mode temporal eigenfunction identifies a rotation feature of the beach profile over the zero spatial eigenfunction location. Figure 7(b) illustrates a schematic diagram of such nearshore bathymetry variation. The beach profile rotation is based on a pivot point at the cross-shore location of $50 \mathrm{~m}$, which is time-independent. At the initial stage, the temporal eigenfunction takes a positive value which indicates a steep nearshore beach slope (the dashed line in Fig. 7b). With the decrease of temporal eigenfunction values, i.e., erosion in the nearshore area and accretion in the offshore zone, the beach slope becomes mild. In case of a zero temporal eigenfunction value around the time of December 2008 (the solid line in Fig. 7b), the EOF's second mode results do not play any role to the beach profile variation under which the bathymetry variability is decided by the third and other higher modes of EOF analysis. Subsequently, the temporal eigenfunction takes a negative value, which leads to a gentle nearshore beach slope (the dotted line in Fig. 7b). In general, the second mode of the EOF analysis presents an anti-clockwise rotation of the mean nearshore beach profile around the cross-shore location of $50 \mathrm{~m}$, which results a milder beach slope within the study period.

In Fig. 6(e), the third mode spatial eigenfunction of the EOF analysis shows negative values in the middle area and positive values on two sides. It also reveals the uniformity in the longshore direction indicating the nearshore response due to cross-shore processes. As for the temporal eigenfunction distribution in Fig. 6(f), it frequently passes through the zero values and leads to multiple nodal points. Higher modes from the EOF analysis are frequently interpreted as the seasonal variation of the beach changes and the shoreline movement (Winant et al., 1975; Fairley et al., 2009), in which clear seasonal behavior of sand movement between winter and summer can be distinguished. However, it is hard to identify any seasonal feature in Fig. 6(f). Nevertheless, the third mode reveals the cross-shore movement of the longshore bar as schematically illustrated in Fig. 7(c). In case of a positive value of the third temporal eigenfunction, a trough beach profile exist in the middle of the study area and nearshore sands are transported and accumulated at both onshore and offshore areas. Sand accumulation to the offshore is primary where the spatial eigenfunction takes large values. As a result, the longshore bar moves to offshore (solid line in Fig. 7c), which corresponds to storm wave conditions. On the contrary, if the temporal eigenfunction takes a negative value, a longshore bar forms at a cross-shore location of $60 \mathrm{~m}$ which is ascribed to the cross-shore sand convergence from both the offshore and onshore regions (dashed line in Fig. 7c) resulted from mild wave condtions. It is found that absolute values of the temporal eigenfunction are commonly larger for positive case than for negative case in Fig. 6(f), which indicates a larger configuration/dimension of the longshore bar located in the offshore region than that of longshore bar existing in the nearshore area (Fig. 7c).

Figure 7(d) presents the original bathymetry data derived from video imagery and the results from EOF analysis. Taking into account the first three modes, the EOF analysis can reproduce the original nearshore bathymetry characteristics fairly well. After the mean profile form the first mode of the EOF analysis, the second and third modes represent more than half of the total bathymetry variability as indicated in Table 2. The discrepancy between the original data and the combined first three EOF mode results is ascribed to the bathymetry variation represented by the higher modes.

Figure 8 shows the higher mode results from the EOF analysis, i.e., forth, fifth and the sixth modes. In case of spatial eigenfunctions, the higher modes show bathymetry variation in the longshore direction, which reveal the typical nearshore response due to longshore processes. Temporal eigenfunctions of the higher modes have values fluctuating around zero, leading to in a number of nodal points. This illuminates the longshore sediment movement frequently changes its direction in this region as also argued in Liu et al. (2011). 

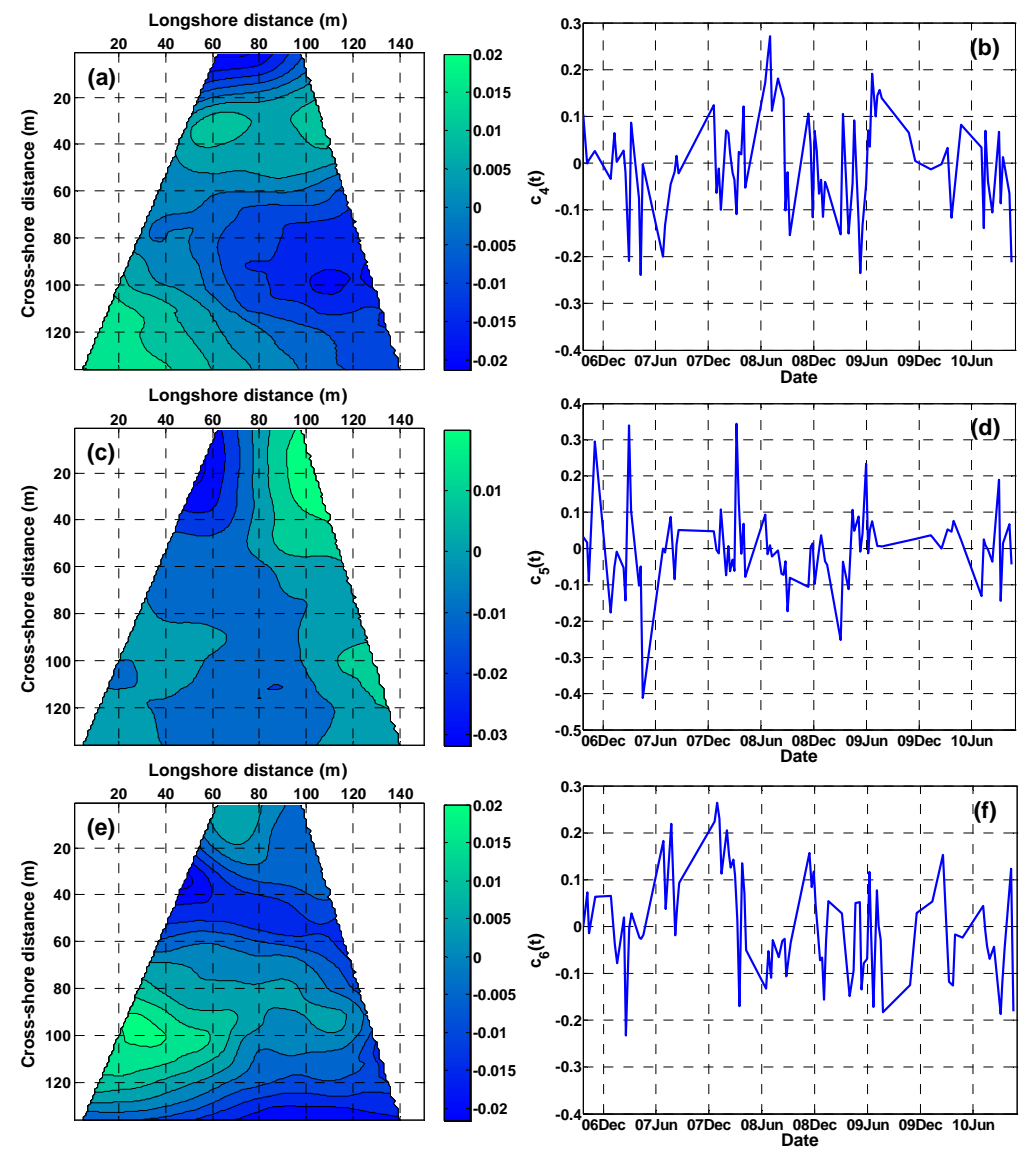

Figure 8. Forth (upper panel), fifth (middle panel) and sixth (lower panel) mode results of the EOF analysis. Left panel: Contour plot of the spatial eigenfunctions; Right panel: temporal eigenfunctions.

\section{CONCLUSIONS}

In this study, a long-term evaluation of the nearshore morphology based on the video imagery was conducted in the Ooita coast, Miyazaki Prefecture, Japan, which covers a time period from September 2006 to December 2010. An automatic nearshore bathymetry mapping technique from the image observation was developed. Various methods were applied to achieve the bathymetry data from image analysis under the long-term duration and the uncontrolled field condition. A flexible approach was introduced for the camera physical calibration to determine the various intrinsic parameters. Wave phase velocity was estimated on the basis of the cross-correlation of the brightness intensity time series of four neighboring horizontal and vertical pixels. Nearshore bathymetry was then estimated from the linear wave dispersion relationship. Data estimation was carried out at the low and high tide levels of each day and integrated in a 7-day period which includes both neap and spring tides. Model validation shows the present automatic bathymetry mapping technique reproduced the ground truth data fairly well with a bias generally less than $0.3 \mathrm{~m}$. The estimated profile is prone to an unrealistic deepening near the shoreline with a maximum overestimation being $0.7 \mathrm{~m}$ due to the omission of wave nonlinearity and wave set-up near the shoreline. Applying this technique, 88 bathymetry data were achieved within the four-year study period. Comparing to the traditional annually-based measurement, it significantly improves the spatiotemporal resolution on the nearshore bathymetry survey.

Subsequently, the two dimensional Empirical Orthogonal Function (EOF) analysis was applied to the time series of nearshore bathymetry data derived from the video imagery in order to evaluate their long-term morphological behaviors. The first three modes of the EOF analysis represent the bathymetry variation from the nearshore response due to the cross-shore process. In which, the first mode reveals the coastal erosion occurred during the study period with a net loss of sand volume in the target region. Erosion was severe before December 2008, especially in the offshore region, and an equilibrium stage was formed afterwards. The second mode shows the mean beach profile rotated in an anti-clockwise direction around a cross-shore location of $50 \mathrm{~m}$, which resulted a milder beach slope in 
the study period. The third mode represents the cross-shore movement of the longshore bar position. The following higher modes of the EOF analysis, such as fourth, fifth and sixth modes, correspond to the bathymetry variation due to the longshore sand movement which frequently changed its direction as indicated from quite a few nodal points in the relevant temporal eigenfunction distributions.

\section{ACKNOWLEDGEMENTS}

The field-recorded image data were provided by the Miyazaki River and Highway Office, Ministry of Land, Infrastructure, Transport and Tourism, Japan.

\section{REFERENCES}

Aarninkhof, S.G.J., Turner, I.L., Dronkers, T.D.T., Caljouw, M., Nipius, L., 2003. A video-based technique for mapping intertidal beach bathymetry. Coastal Eng., 49, 275-289.

Aarninkhof, S.G.J., Ruessink, B.G., Roelvink, J.A., 2005. Nearshore subtidal bathymetry from timeexposure video images. J. Geophys. Res., 110, C06011, doi: 10.1029/2004JC002791.

Aubrey, D.G. 1979. Seasonal patterns of onshore/offshore sediment movement. J. Geophys. Res., 84(C10), 6347-6354.

Bell, P.S., 1999. Shallow water bathymetry derived from an analysis of X-band marine radar images of waves. Coastal Eng., 37, 513-527.

Clarke, L.B., Werner, B.T., 2003. Synoptic imaging of nearshore bathymetric patterns. J. Geophys. Res., 108(C1), 3005, doi: 10.1029/2000JC000780.

Davidson, M.A., Turner, I.L., 2009. A behavioral template beach profile model for predicting seasonal to interannual shoreline evolution. J. Geophys. Res., 114, F01020, doi: 10.1029/2007JF000888.

Dongeren, A.V., Plant, N., Cohen, A., Roelvink, D., Haller, M.C., Catalan, P., 2008. Beach Wizard: Nearshore bathymetry estimation through assimilation of model computations and remote observations. Coastal Eng., 55, 1016-1027.

Fairley I., M. Davidson, K. Kingston, T. Dolphin, and R. Phillips. 2009. Empirical orthogonal function analysis of shoreline changes behind two different designs of detached breakwaters. Coastal Eng., 56(11-12), 1097-1108.

Guillen, J., Garcia-Olivares, A., Ojeda, E., Osorio, A., Chic O., Gonzalez R., 2008. Long-term quantification of Beach users using video monitoring. J. Coastal Res., 24(6), 1612-1619.

Heikkila, J., Silven, O., 1997. A four-step camera calibration procedure with implicit image correction. IEEE Computer Society Conf. on Computer Vision and Pattern Recognition (CVPR'97), San Juan, Puerto Rico, 1106-1112.

Holland, K.T., Holman, R.A., Lippmann, T.C., Stanley, J., 1997. Practical use of video imagery in nearshore oceanographic field studies. IEEE J. of Oceanic Eng., 22(1), 81-92.

Holman, R.A., and Stanley. J., 2007. The history and technical capabilities of Argus. Coastal Eng., 54(6-7), 477-491.

Horikawa, K., Kuo, C.-T., 1966. A study on wave transformation inside surf zone. Proceedings of 10th International Conference on Coastal Engineering, ICCE1966, Tokyo, Japan, 217-233.

Hsu, T.-W., Ou, S.-H., Wang, S.-K., 1994. On the prediction of beach changes by a new 2-D empirical eigenfunction model. Coastal Eng., 23, 255-270.

Jimenez, J.A., Osorio, A., Marino-Tapia, I., Davidson, M., Medina, R., Kroon, A., Archetti, R., Ciavola, P., Aarnikhof, S., 2007. Beach recreation planning using video-derived coastal state indicators. Coastal Eng., 54(6-7), 507-521.

Kanno, A., Koibuchi, Y., Isobe, M., 2011. Statistical combination of spatial interpolation and multispectral remote sensing for shallow water bathymetry. IEEE Geoscience and Remote Sensing Letters, 8(1), 64-67.

Kroon, A., Davidson, M.A., Aarninkhof, S.G.J., Archetti, R., Armaroli, C., Gonzalez, M., Medri, S., Osorio, A., Aagaard, T., Holman, R.A., Spanhoff, R., 2007. Application of remote sensing video systems to coastline management problems. Coastal Eng., 54(6-7), 493-505.

Larson, M., Capobianco, M., Jansen, H., Rozynski, G., Southgate, H., Stive, M., Wijnberg, K.M., Hulscher, S., 2003. Analysis and modeling of field data on coastal morphological evolution over yearly and decadal time scales. Part 1: Background and linear techniques. J. Coastal Res., 19(4), 760-775.

Lippmann, T.C., Holman, R.A., 1989. Quantification of sand bar morphology: A video technique on wave dissipation. J. Geophys. Res., 94 (C1), 995-1011. 
Liu, H., Tajima, Y., Sato, S., 2008. Field study on the nearshore sediment process around the Tenryu Estuary using image analysis. Proceedings of 31st International Conference on Coastal Engineering, ICCE2008, Hamburg, Germany, 2064-2076.

Liu, H., Funayama, H., Tajima, Y., Sato, S., 2009. Quantification of in situ coastal surfers based on video monitoring. Proceedings of the 5th Asian and Pacific Coastal Engineering Conference, APAC2009, Singapore, Vol. 2, 90-97.

Liu, H., Tajima, Y., Sato, S., 2010. Long-term monitoring on the sand spit morphodynamics at the Tenryu River Mouth. Proceedings of 32nd International Conference on Coastal Engineering, No. 32, ICCE2010, Shanghai, China.

Liu, H., Hamamoto, A., Sato, S., 2011. Monitoring the nourished sand longshore movement based on feldspar luminescence measurement. Proceedings of Coastal sediments'11, Miami, Florida, USA, Vol. 1, 57-70.

Lyzenga, D.R., 1985. Shallow-water bathymetry using combined LIDAR and passive multispectral scanner data. Int. J. Remote Sens., 6(1), 115-125.

Miller J.K., and R.G. Dean. 2007. Shoreline variability via empirical orthogonal function analysis: Part I temporal and spatial characteristics. Coastal Eng., 54(2), 111-131.

Pearre, N.S., Puleo, J.A., 2009. Quantifying seasonal shoreline variability at Rehoboth Beach, Delaware, using automated imaging techniques. J. Coastal Res., 25(4), 900-914.

Plant, N.G., Holman, R.A., 1997. Intertidal beach profile estimation using video images. Marine Geology, 140, 1-24.

Plant, N.G., Holland, K.T., 2011. Prediction and assimilation of surf-zone processes using a Bayesian network Part I: Forward models. Coastal Eng., 58, 119-130.

Pruszak, Z., 1993. The analysis of beach profile changes using Dean's method and empirical orthogonal functions. Coastal Eng., 19, 245-261.

Reeve, D.E., Horrillo-Caraballo, J.M., Magar, V., 2008. Statistical analysis and forecasts of long-term sandbank evolution at Great Yarmouth, UK. Estuarine, Coastal and Shelf Science, 79, 387-399.

Reeve, D., Li, B., Thurston, N., 2001. Eigenfunction analysis of decadal fluctuations in sandbank morphology at Gt Yarmouth. J. Coastal Res., 17(2), 371-382.

Ruessink, B.G., van Enckevort, I.M.J., Kuriyama, Y., 2004. Non-linear principal component analysis of nearshore bathymetry. Marine Geology, 203, 185-197.

Smit, M.W.J., Aarninkhof, S.G.J., Wijnberg, K.M., Gonzalez, M., Kingston, K.S., Southgate, H.N., Ruessink, B.G., Holman, R.A., Siegle, E., Davidson, M., Medina, R., 2007. The role of video imagery in predicting daily to monthly coastal evolution. Coastal Eng., 54(6-7), 539-553.

Smith, R.K., Bryan, K.R., 2007. Monitoring beach face volume with a combination of intermittent profiling and video imagery. J. Coastal Res., 23(4), 892-898.

Southgate, H.N., Wijnberg, K.M., Larson, M., Capobianco, M., Jansen, H., 2003. Analysis of field data of coastal morphological evolution over yearly and decadal timescales. Part 2: Non-linear techniques. J. Coastal Res., 19(4), 776-789.

Stockdon, H.F., Holman, R.A., 2000. Estimation of wave phase speed and nearshore bathymetry from video imagery. J. Geophys. Res., 105 (C9), 22015-22033.

Takewaka, S., 2005. Measurements of shoreline positions and intertidal foreshore slopes with X-band marine radar system. Coastal Eng. J., 47(2-3), 91-107.

Thornton, E.B., Guza, R.T., 1982. Energy saturation and phase speeds measured on a natural beach. $J$. Geophys. Res., 87(C12), 9499-9508.

Uda, T., Hashimoto, H., 1982. Description of beach changes using an empirical predictive model of beach profile changes. Proceedings of 18th International Conference on Coastal Engineering, Cape Town, South Africa, 1405-1418.

Uunk, L., Wijnberg, K.M., Morelissen, R., 2010. Automated mapping of the intertidal beach bathymetry from video images. Coastal Eng., 57, 461-469.

Van Enckevort, I.M.J., Ruessink, B.G., 2001. Effect of hydrodynamics and bathymetry on video estimates of nearshore sandbar position. J. Geophys. Res., 106(C8), 16969-16979.

Winant, C.D., D.L. Inman, and C.E. Nordstrom. 1975. Description of seasonal beach changes using empirical eigenfunctions. J. Geophys. Res., 80(15), 1979-1986.

Zhang, Z., 2000. A flexible new technique for camera calibration. IEEE Transactions on Pattern

Analysis and Machine Intelligence, 22(11), 1330-1334. 